\title{
Alternative Mechanisms for Gaia
}

\author{
Arwen E. Nicholson*1, David M. Wilkinson ${ }^{2}$, Hywel T.P. Williams ${ }^{1}$, Timothy \\ M. Lenton ${ }^{1}$ \\ ${ }^{1}$ Earth System Science, University of Exeter, Streatham Campus, Exeter, EX4 4PY, UK \\ ${ }^{2}$ School of Life Sciences, University of Lincoln, Joseph Banks Laboratories, Green Lane, \\ Lincoln, LN6 7DL, UK
}

\begin{abstract}
A long-standing objection to the Gaia hypothesis has been a perceived lack of plausible mechanisms by which life on Earth could come to regulate its abiotic environment. A null hypothesis is survival by pure chance, by which any appearance of regulation on Earth is illusory and the persistence of life simply reflects the weak anthropic principle - it must have occurred for intelligent observers to ask the question. Recent work has proposed that persistence alone increases the chance that a biosphere will acquire further persistence-enhancing properties. Here we use a simple quantitative model to show that such 'selection by survival alone' can indeed increase the probability that a biosphere will persist in the future, relative to a baseline of pure chance. Adding environmental feedback to this model shows either an increased or decreased survival probability depending on the initial conditions. Feedback can hinder early life becoming established if initial conditions are poor, but feedback can also prevent systems from diverging too far from optimum environmental conditions and thus increase survival rates. The outstanding question remains the relative importance of each mechanism for the historical and continued persistence of life on Earth.
\end{abstract}

*arwen.e.nicholson@gmail.com

Preprint submitted to Elsevier

August 10, 2018 
The Gaia hypothesis postulates that life on Earth forms part of a selfregulating planetary-scale system with stabilising properties that help to maintain habitable conditions [1, 2]. Early critiques of Gaia by evolutionary biologists questioned the compatibility of Gaia with natural selection [3, 4] and noted that an appeal to the weak anthropic principle could account for the long persistence of life on Earth without requiring regulatory mechanisms [3]. Subsequent proposals that global environmental feedbacks could be built on by-products of metabolic traits selected for more proximate ecological benefits sidestepped this evolutionary critique, but raised the question of why stable outcomes would be any more likely than unstable ones [5, 6, 7. A series of theoretical models of feedback between life and the environment then showed that self-stabilising outcomes can arise from metabolic by-products in a manner consistent with natural selection [8, 9, 10] and possible selection mechanisms for Gaia have been identified across multiple scales [11.

Recently, a new schematic model of 'selection by survival' alone has been proposed [12, whereby postulated biospheres can acquire persistence-enhancing adaptations by chance over time. In the language of this model, macro-level 'mutations' affecting biosphere dynamics and stability arise due to micro-level mutations that occur during reproduction of the organisms that compose the system. Thus the longer the biosphere persists, the greater the likelihood that persistence-enhancing mutations can arise. This could apply to the Earth's biosphere as well as smaller entities such as ecosystems (see [13] for a wider discussion of such approaches). Here we introduce a quantitative model of this idea and contrast it with a null model of survival by pure chance, and then investigate the effect of adding feedback using an existing model of environmental feedbacks.

\section{Model}

We compare three hypotheses for the continued persistence of life on Earth: H1 - Null hypothesis (pure chance) 

44 is a constant:

$$
P_{C}=C
$$

45

H2 - Acquisition of persistence enhancing mutations by chance (selection by survival)

H3 - Environmental feedbacks in addition to selection by survival.

In an attempt to isolate the effect of life on its own persistence we use idealised model biospheres where the abiotic environment is highly simplified and where the biosphere has a non-zero likelihood of extinction which can be impacted by the biosphere. For each scenario, we consider a non-interacting population of $10^{4}$ isolated model biospheres and consider how many survive as a function of time. Appendix A contains a full model description. The models used in each scenario are described below.

\subsection{H1 - Null Hypothesis}

For the null model we assume a constant extinction probability for each biosphere at each model timestep, resulting in exponential decay in the number of surviving biospheres. The probability of extinction at any time for H1 systems

We set $C$ to have the same value as the starting extinction values for the selection by survival (H2), and the environmental feedback (H3) experiments. This allows us to identify any survival enhancement performed by the biospheres in these experiments.

\subsection{H2 - Selection by Survival}

For the selection by survival [12] experiment we adapt a pre-existing model - the Flask model [8, 9, 10, 14]. This consists of model 'flasks', host to microbe communities (these could be thought of as effectively chemostats - at the small scale - to whole biospheres, at a large scale). These flasks experience inflow and outflow of a medium containing nutrients. Microbes consume nutrients, produce waste, and impact the abiotic environment (here represented as a single variable 
arbitrarily labelled 'temperature', $T$ ) as a by-product of their metabolic activity. The flask inflow medium is at a constant temperature $T_{\text {inflow }}$. Microbes starve to death if their biomass drops below a certain threshold $B_{D}$ and reproduce asexually if their biomass reaches the reproduction threshold $B_{R}$. During reproduction mutations can occur leading to new species emerging. There is also a constant probability of random mortality of microbes $D$. There are 4 nutrient types in each system, and microbes can consume and excrete a combination of any, however every microbe must both consume and excrete, and it must not consume what it excretes, otherwise it is unviable. The pattern of consumption / excretion is set by the microbe's genotype, which can mutate at reproduction.

For our selection by survival experiments, we limit the system to a single flask; we can think of these flasks as self-contained planets host to biospheres. Microbial metabolisms impact the system temperature, but this temperature does not impact individual microbes' metabolisms. The temperature does impact the biosphere-wide probability of extinction $P_{T}$ :

$$
P_{T}=a+b \times\left|T_{\text {ideal }}-T\right|
$$

where $T$ is the system temperature, and depends on the genetic makeup of the microbe community currently alive in the system, $a=2 \times 10^{-6}$ is a constant background probability of global extinction, and $b=2 \times 10^{-6}$ controls the impact the system temperature has on the probability of extinction. Here there is a global $T_{\text {ideal }}$ value, which is the system temperature that results in the lowest probability of total extinction. The inflow to the system is at temperature $T_{\text {inflow }} \ll T_{\text {ideal }}$. This makes initial conditions far from ideal, however still tolerable to the temperature sensitive H3 microbes, thus allowing for direct comparison. We seed with a single microbe species set to have no impact on the system temperature. As mutants arise, they will affect system temperatures via their metabolisms, but for each experiment the environment starts with the same conditions. This allows us to see the effect the selection by survival mechanism has more clearly. The selection by survival model is very similar 
to the version of Flask model detailed in [14 however with the feedback from environment to microbe metabolisms removed, and the temperature dependant $P_{T}$ - the biosphere-level mortality process - imposed on each system.

\subsection{H3 - Adding Environmental Feedbacks}

The environmental feedback case (H3) is similar to the selection by survival model (H2) with the key difference that the temperature does now impact individual microbes' growth rates. H2 systems have feedback acting in one direction only, from the microbes to the environment. H3 systems have the same life $\rightarrow$ environment interaction, but also feedback from the environment to the microbes, thus closing the feedback loop.

For H3 systems, microbes are temperature sensitive with their growth rate impacted by the system temperature. The growth rates for all microbes are at a maximum when $T=T_{\text {ideal }}$, i.e. $T_{\text {ideal }}$ is the temperature at which their metabolic activity will be at its peak. As the system temperature moves away from $T_{i d e a l}$, microbe metabolic activity slows until eventually they cannot consume nutrients at all. If conditions do not quickly improve, the result is individual mortality of microbes which can lead to extinction. This model is the Flask model described in [14], however with the biosphere-level mortality function $P_{T}$ (Equation 2) imposed on each system. The microbes' temperature sensitivity is determined by a parameter $\tau$ that takes a real positive value, and is the same for all species (earlier work e.g. [10] explores scenarios where $\tau$ differs between species). A higher $\tau$ value corresponds to more temperature sensitive microbes. The fitness $F$ of the microbes depends on $\tau$ in the following way:

$$
F=e^{-\left(\tau\left|T_{\text {ideal }}-T\right|\right)^{2}}
$$

Setting $\tau=0$ would give a system of microbes that are completely temperature insensitive with $F=1$ for all temperatures, i.e. H2 systems. When $\tau>0$, the microbes' fitness is a Gaussian function, centred around $T=T_{\text {ideal }}$, and as $\tau \rightarrow \infty$, the fitness function becomes a delta function, with a non-zero value for only $T=T_{\text {ideal }}$. When the microbes metabolisms are at a maximum, 
the system will also have the lowest $P_{T}$ value (as determined by Equation 2 ).

For H3 systems, microbes now feel the effects of an improving or a degrading environment and their metabolic activity will be impacted - this in turn will impact the system temperature resulting in a feedback loop.

Figure 1 shows a schematic of an H2 / H3 system. It is these systems that are subject to the $P_{T}$ extinction values, and the microbial actions happening inside each system determine the value of $P_{T}$.
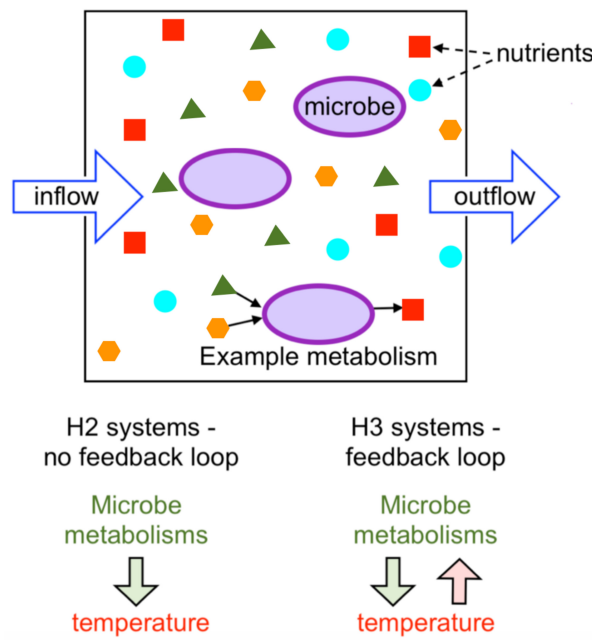

Figure 1: Schematic of an H2 or H3 system, showing the nutrient medium (with inflow and outflow), the microbes, and an example microbe metabolism. For H2 systems, microbe metabolisms impact the system temperature, but are temperature independent themselves. In H3 systems, microbe metabolisms impact the system temperature and are temperature sensitive, resulting in a feedback loop.

\section{Method}

We start the experiment with each model system with a temperature that does not match the microbes 'ideal' temperature (i.e. with $T_{0} \neq T_{\text {ideal }}$ ) to test the model's ability to approach ideal conditions. All H1, H2, and H3 systems begin each experiment with the same system temperature, $T_{0}$, and we set $P_{C}=$ $P_{T, 0}=a+b \times\left|T_{\text {ideal }}-T_{0}\right|$. The value of $T_{0}$ will be the temperature of the medium 
inflowing to each system, $T_{\text {inflow }}$. We set $T_{\text {inflow }}=0$, and therefore $T_{0}=0$, for all experiments. For all experiments, the parameters $a$ and $b$ in Equation 2 have the values $a=b=2 \times 10^{-6}$. The values of $P_{C}$ vary as $T_{i d e a l}$ varies between experiments and we have $P_{C}=\left\{10.02 \times 10^{-4}, 6.02 \times 10^{-4}, 2.02 \times 10^{-4}\right\}$ for the corresponding values of $T_{\text {ideal }}=\{500,300,100\}$.

H2 and H3 systems are seeded with a single microbe species with a metabolism that has zero impact on the system temperature. When mutants emerge, their metabolisms may impact the temperature. We perform $10^{4}$ experiments with different random initialisations for each hypothesis in order to robustly observe the system behaviours exhibited in each scenario.

To test the H2 hypothesis we perform 3 sets of experiments for systems with $T_{\text {ideal }} \in\{500,300,100\}$. To then test how H3 systems compare to H2, we perform, for each $T_{\text {ideal }}$ case, 3 further studies with differing $\tau$ strengths to investigate how changing the microbes' sensitivity impacts model results. For $T_{i d e a l}=500$ we perform H3 experiments with $\tau \in\{0.002,0.0025,0.003\}$, for $T_{i d e a l}=300$ we investigate $\tau \in\{0.003,0.004,0.005\}$, and for $T_{i d e a l}=100$, our H3 experiments are $\tau \in\{0.005,0.007,0.009\}$. This allows us to explore how these key parameters impact the system behaviours. The starting $\tau$ value for each $T_{\text {ideal }}$ set of experiments corresponds to the end $\tau$ for the previous set. I.e. the last $\tau$ explored for $T_{\text {ideal }}=500$ is $\tau=0.003$ and thus the first $\tau$ explored for $T_{\text {ideal }}=300$ is also $\tau=0.003$. This allows us to see how shifting $T_{\text {ideal }}$ while keeping $\tau$ constant affects the H3 systems while still enabling us to explore a suitable $\tau$ range for each $T_{\text {ideal }}$. An in-depth exploration of how various system parameters impact the Flask model can be found in $[8,9,10,14$.

\section{Results}

How the $\mathrm{H} 2$ and $\mathrm{H} 3$ experiments perform, compared to each other and compared to the null, is strongly dependent on how closely the initial conditions match the microbes' preferred conditions, i.e. how large the value of $\left|T_{\text {ideal }}-T_{0}\right|$ is, and for H3 systems, how sensitive the microbes are to their environment. 
This is summarised in Figure 5, which shows a series of experiments with varying $T_{\text {ideal }}$ values and microbe sensitivities $\tau$ (with $T_{0}=0$ for each case). These figures show the number of surviving systems over time for the null H1 systems, selection by survival H2 systems, and environmental feedback H3 systems. Each figure also includes the 'Ideal' survival probability i.e. for $\left|T_{\text {ideal }}-T\right|=0$ for all time. The 'Ideal' case is included as a 'perfect world' baseline for comparison. We return to this part of the analysis later, after first considering the behaviours of the H1, H2, and H3 systems.

\subsection{H2 systems (selection by survival)}

We first examined individual H2 systems to determine their typical characteristics. H2 systems are not temperature sensitive and so the only limiting factor on the total population size is nutrient availability. Once the population has reached the carrying capacity of the environment (i.e. consuming all available nutrients) the population will remain stable there, regardless of the system temperature. The system temperature will change as the microbe community changes; as new mutants emerge, species die, and the relative populations of existing species shift, the temperature will perform a 'random walk' and change in an unguided manner.

Figure 2 shows the trajectories of the system temperature (with $T_{0}$ shown in black and $T_{\text {ideal }}$ shown in green) and the total population for 3 individual runs. System 1 (Figures 2a and 2d) shows several abrupt temperature changes happening in the system, and these correspond with jumps in the total population, shown in Figure 2d, Each system is seeded with a single microbe species that consumes only 1 nutrient source, therefore at the beginning of each experiment, there are 3 unexploited sources of food (as there are 4 nutrient types in total). If a mutant emerges that consumes a currently abundant nutrient, its population can rapidly increase due to the abundance of food, causing rapid temperature changes due to the metabolic byproducts of this new mutant. Once all nutrients are exploited, new mutants emerging have no advantage over existing species and so temperature changes can remain relatively stable over long time periods. 


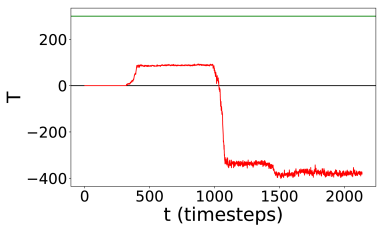

(a) System 1

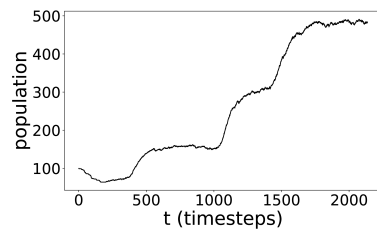

(d) System 1

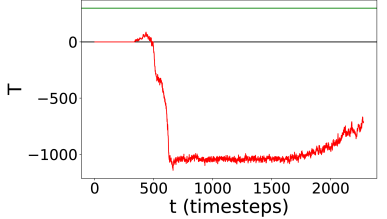

(b) System 2

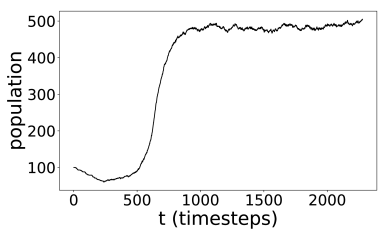

(e) System 2



(c) System 3

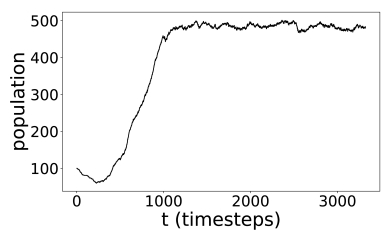

(f) System 3

Figure 2: Temperature (T) vs time and total population vs time for individual H2 systems with $T_{\text {ideal }}=300$. In the temperature plots $T_{0}$ is shown as a black horizontal line, and $T_{i d e a l}$ is shown in green.

To test whether selection by survival is a viable mechanism, we first examine the $\mathrm{H} 2$ systems and compare them to the null H1 systems. Figure 3 shows the surviving number of $\mathrm{H} 2$ biospheres (red lines) and $\mathrm{H} 1$ biospheres (black solid lines) over time, where $T_{\text {ideal }} \in\{500,300,100\}$ respectively (note the log-scale $\mathrm{y}$-axis). Figure $3 \mathrm{a}$ shows the results for the experiments where $T_{\text {ideal }}=500$ and initial conditions are far from $T_{\text {ideal }}\left(T_{0}=0\right)$. Initially the $\mathrm{H} 2$ and $\mathrm{H} 1$ systems die out at a similar rate, but after this initial period the $\mathrm{H} 2$ systems begin to show improved persistence relative to the $\mathrm{H} 1$ systems, and a significant number of $\mathrm{H} 2$ biospheres go on to live for much longer timespans than the longest lived 
H1 system.

For H1 systems, the biosphere does not impact the system temperature, $T$, and therefore $P_{C}$, is constant for all time. In $\mathrm{H} 2$ systems, microbes are changing their environment as they metabolise nutrients and this can either increase or decrease their survival probability. For those that degrade their environment, their $P_{T}$ value will be higher than the $P_{C}$ value, and hence these degrading systems tend to go extinct faster. However, those that improve their environment experience lower $P_{T}$ values and so can experience much longer lifespans.

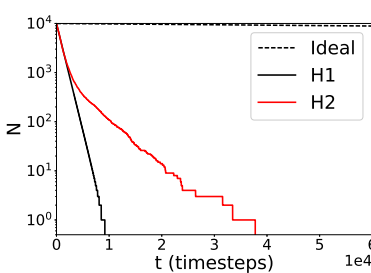

(a) $T_{\text {ideal }}=500$

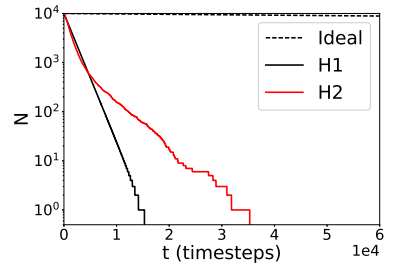

(b) $T_{\text {ideal }}=300$

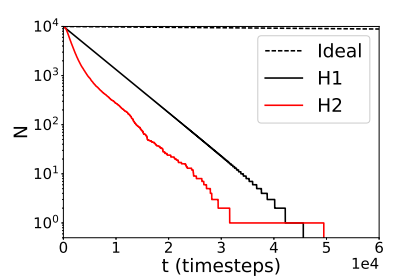

(c) $T_{\text {ideal }}=100$

Figure 3: Number of surviving biospheres (N) against time. Selection by survival systems (H2) shown in red, and null hypothesis (H1) in black. Note the log-scale y-axis.

Figures $3 \mathrm{~b}$ and $3 \mathrm{c}$ show that as $T_{\text {ideal }}$ comes closer to $T_{0}$, the positive impact of selection by survival decreases, until H2 systems suffer on average poorer survival rates than $\mathrm{H} 1$ systems. Each $\mathrm{H} 2$ biosphere is effectively a randomly walking system, with the impact from the microbes on the environment changing as the microbe community changes over time due to death, reproduction and mutation. When initial conditions are far from ideal, there are a large number of possible random walks that will improve the environment and thus improve survival odds. As the distance between $T_{\text {ideal }}$ and $T_{0}$ closes, the number of random walks that are environment improving decreases, until, for conditions where $T_{i d e a l}=T_{0}$, any alteration of the environment by the microbes decreases survival probabilities. The case where $T_{\text {ideal }}=T_{0}=T$ for all time is shown in each figure as the 'Ideal' case. Therefore the mechanism of selection by survival sees the best improvement in biosphere survival probability when initial 
conditions are poor for life.

Although the relative success between $\mathrm{H} 1$ and $\mathrm{H} 2$ systems is impacted by changing $T_{\text {ideal }}$, the $\mathrm{H} 2$ survival rates in Figures $3 \mathrm{a}$ - $3 \mathrm{c}$ are similar - the distance between $T_{\text {ideal }}$ and $T_{0}$ does not greatly impact the selection by survival mechanism. For different $T_{\text {ideal }}, \mathrm{H} 2$ systems start with different $P_{T, 0}$ values, however as $\mathrm{H} 2$ biospheres rapidly move the system temperature away from $T_{0}$ and keep it under their control, the starting proximity to ideal environmental conditions ceases to matter. For H1 systems where $T=T_{0}$ for all time, the distance between $T_{\text {ideal }}$ and $T_{0}$ has a large impact on survival rates.

\subsection{H3 systems (environmental feedback)}

H3 microbes, in constrast to $\mathrm{H} 2$ microbes, are temperature sensitive and so changes in the system temperature impact their metabolisms. Figure 4 shows the temperature and total population for the individual trajectories of $3 \mathrm{H} 3$ systems, with $T_{0}$ shown in black and $T_{\text {ideal }}$ in green. Some H3 systems behave similarly to $\mathrm{H} 2$ systems, with the total population quickly reaching the carrying capacity, and the temperature slowly changing as the microbe community changes, e.g. System 1 (Figures $4 \mathrm{a}$ and $4 \mathrm{~d}$ ). For systems where the temperature wanders towards the bounds of habitability, H3 systems behave differently. The temperatures in both Systems 2 and 3 (Figures $4 \mathrm{~b}$ and $4 \mathrm{c}$ ) wander far from $T_{i d e a l}$ and then remain at a near constant temperature. In System 2, while the temperature remains near constant, the population changes over time (Figure 4e), and in System 3, the population remains at a near stable population lower than the carrying capacity, and lower than previous populations experienced by the system (Figure 4f). In these cases the H3 systems enter temperature limiting regimes, with System 2 entering the lower temperature limiting regime at $T=T_{\text {lim }}^{-} \approx-100$ at $t \approx 1900$ and System 3 entering the high temperature limiting regime, with $T=T_{\text {lim }}^{+} \approx 700$, at $t \approx 2000$.

These temperature limiting regimes are characterised by a near stable temperature maintained by a negative feedback loop, with the total impact of the biosphere on the environment 'pulling' the system temperature one way, and 
the inflow medium at $T=T_{0}$ 'pulling' the temperature in the opposite direction. This is known as 'single rein-control' [14. For a system to be at $T=T_{\text {lim }}^{+}$ the biosphere must be overall heating, and similarly for $T=T_{\text {lim }}^{-}$the biosphere must be overall cooling. At $T=T_{\text {lim }}^{+/-}$if the environment degrades, the total population reduces as microbe metabolisms suffer, and this reduces the cause of the environmental degradation (microbe metabolic byproducts) allowing the inflow medium to bring temperatures back towards $T_{0}$ thus improving habitability. If at $T_{\text {lim }}^{+/-}$, the environment improves, microbes will proliferate, increasing their impact on their environment and pushing temperatures back towards $T=T_{\text {lim }}^{+/-}$. This leads to temperature regulation, which can be exited via a positive feedback (microbes causing environment improvement and leading to greater populations) until either the alternate temperature limiting regime is reached, or the system becomes nutrient limited. System 2 (Figure 4e clearly shows the microbe population adjusting in response to microbe community changes while a near constant temperature is maintained (Figure 4b).

The values of $T_{\text {lim }}^{+}$and $T_{\text {lim }}^{-}$will depend on $\tau$ and $T_{\text {ideal }}$. A higher $\tau$ will result in a smaller distance between $T_{\text {ideal }}$ and $T_{\text {lim }}^{+/-}$. Examining Figure $4 \mathrm{~b}$ we can see that for a strong enough $\tau, T_{\text {lim }}^{-}>T_{0}$ would be true. If this were the case, the initial microbe population would be unable to survive its environment and would quickly go extinct. If $T_{l i m}^{-} \approx T_{0}$, then the early environment will only be able to support a very small cooling biosphere which would be more prone to extinction due to stochastic fluctuations. Biospheres with high $\tau$ will also have a narrower window of temperatures where the system is nutrient limited and so these systems will be more likely to become temperature limited and become 'stuck' at these $T_{\text {lim }}^{+/-}$values, which would prevent the temperature from reaching values closer to $T_{i d e a l}$ and thus prevent the corresponding low $P_{T}$ values for H3 systems.

We ran similar survival experiments to those shown in Figure 3 with H3 systems (see Figure 5). Whether the added feedback from the environment to the microbes helps or hinders an H3 biosphere's survival probability depends on how far the starting conditions are from $T_{\text {ideal }}$, and the value of $\tau$. H2 biospheres 


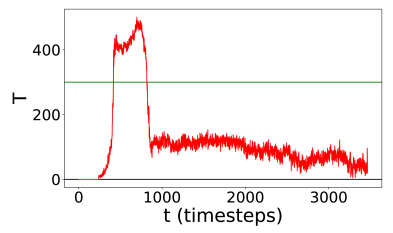

(a) System 1

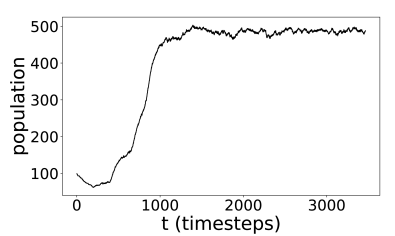

(d) System 1

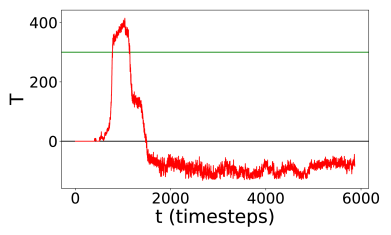

(b) System 2

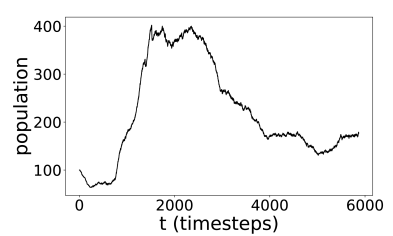

(e) System 2

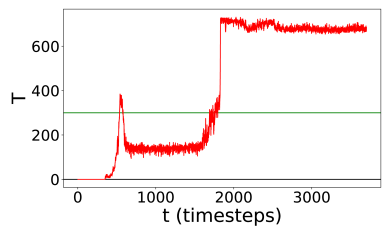

(c) System 3

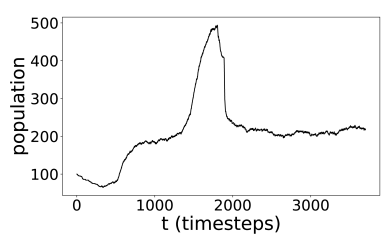

(f) System 3

Figure 4: Temperature vs time and total population vs time for individual H3 systems with $T_{i d e a l}=300$ and $\tau=0.003$. In the temperature figures $T_{0}$ is shown as a black horizontal line, and $T_{\text {ideal }}$ is shown in green.

can be though of as a limiting case of H3 biospheres with microbe sensitivity $\tau=0$. Figure 5 shows the results of experiments with varying $T_{i d e a l}$, and $\tau$ values for $\mathrm{H} 1, \mathrm{H} 2, \mathrm{H} 3$ and 'Ideal' systems.

The first column in Figure 5 shows systems where $T_{i d e a l}=500$ and $\tau$ (microbe sensitivity) increases for $\mathrm{H} 3$ biospheres as we move down the column. Figure 5a, with $\tau=0.002$ shows feedback hindering the H3 biospheres' survival rates compared to $\mathrm{H} 2$ systems where $T_{\text {ideal }}=500$. As $\tau$ increases slightly to $\tau=0.0025$ (Figure $5 \mathrm{~d}$ ), the H3 biosphere survival rates are reduced more. Figure $5 \mathrm{~g}$, where $\tau=0.003$, shows that with strong enough feedback H3 systems rapidly go extinct and highlights a feature of $\mathrm{H} 3$ systems that is not present in $\mathrm{H} 2$ systems - extinction via starvation. As H3 microbes are temperature sensitive, if they find their environment too inhospitable they will be unable to consume nutrients and the biosphere will quickly go extinct. In Figure $5 \mathrm{~g}$, microbes are seeded in an environment they cannot tolerate, dooming them to a rapid extinction. Figure 5j] shows the fitness curves for the H3 microbes against temperature for each of the $\tau$ values explored when $T_{\text {ideal }}=500$.

Figure $5 \mathrm{~s}$ second column shows experiments with $T_{\text {ideal }}=300$. Figure $5 \mathrm{~b}$ 

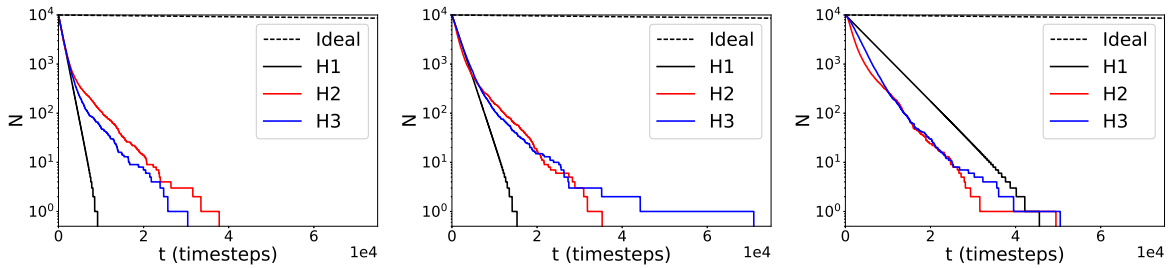

(a) $T_{\text {ideal }}=500 \tau=0.002$

(b) $T_{\text {ideal }}=300 \tau=0.003$

(c) $T_{\text {ideal }}=100 \tau=0.005$
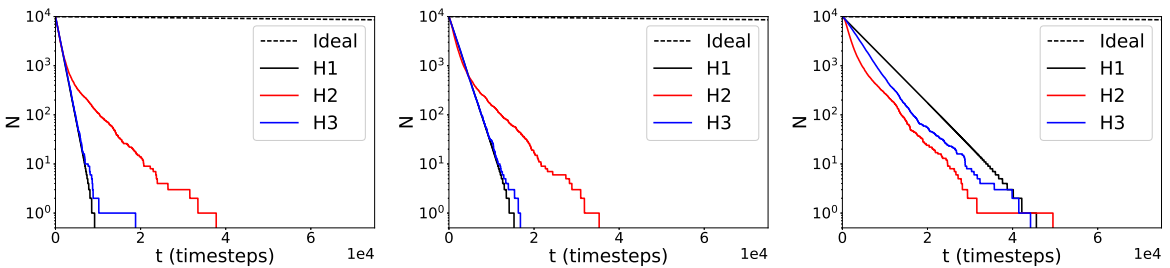

(d) $T_{\text {ideal }}=500 \tau=0.0025$

(e) $T_{\text {ideal }}=300 \tau=0.004$

(f) $T_{\text {ideal }}=100 \tau=0.007$
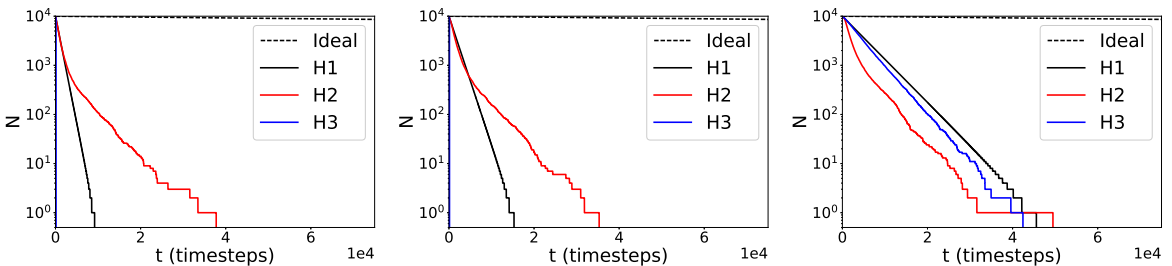

(g) $T_{\text {ideal }}=500 \tau=0.003$

(h) $T_{\text {ideal }}=300 \tau=0.005$

(i) $T_{\text {ideal }}=100 \tau=0.009$
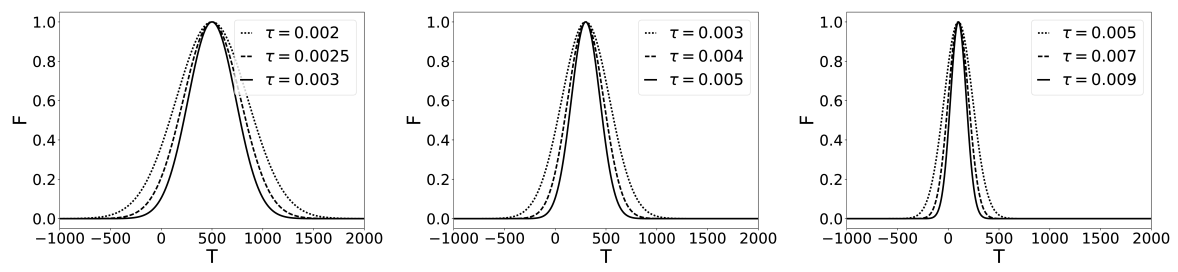

(j) Fitness vs $\mathrm{T}$ for

(k) Fitness vs $\mathrm{T}$ for

(1) Fitness vs $\mathrm{T}$ for

$T_{\text {ideal }}=500$

$T_{\text {ideal }}=300$

$T_{\text {ideal }}=100$

Figure 5: Number of surviving biospheres $(\mathrm{N})$ against time, for experiments with differing $T_{\text {ideal }}$ and $\tau$ (microbe sensitivity) values. For all experiments $T_{0}=0$.

show experiments with $\tau=0.003$ for H3 microbes. Comparing Figures $5 \mathrm{~g}$ and 
few H3 systems to out live all H2 systems. With stronger feedback, 'anti-Gaian' dynamics can be more strongly countered provided the early biosphere is able to survive. Figure $5 \mathrm{e}$ shows that increasing $\tau$, this time in larger increments, again starts to hinder the survival rates of H3 systems as the early biospheres struggle to establish themselves and / or become 'stuck' in the temperature limiting regimes. Figure $5 \mathrm{~h}$ with $\tau=0.005$ shows the H3 systems rapidly going extinct via starvation as the microbes are unable to survive their initial environment. Figure 5k shows the H3 microbes' fitness curves for the $\tau$ values explored for $T_{\text {ideal }}=300$. The widest fitness curve in Figure $5 \mathrm{k}$ corresponds to the narrowest fitness curve in Figure $5 \mathrm{j}$ but shifted to the left as $T_{i d e a l}$ moves closer to $T_{0}=0$.

The third column in Figure 5 shows biospheres with $T_{\text {ideal }}=100$. Here we see that H3 systems overall experience higher survival rates over H2 systems for the range of $\tau$ explored. Comparing Figures $5 \mathrm{~h}$ and $5 \mathrm{c}$ we see that keeping $\tau=0.005$ constant, but moving $T_{\text {ideal }}$ closer to $T_{0}$, the survival rates of $\mathrm{H} 3$ systems are vastly improved, again demonstrating that the positive or negative impact environmental feedback can have on biospheres' survival rates is strongly dependant on the starting environmental conditions. As we increase $\tau$ to $\tau=$ 0.007 (Figure $5 \mathrm{f}$ ) and $\tau=0.009$ (Figure $5 \mathrm{i}$ ) we see that, in the survival rate for H3 systems is increasing - feedback improves H3 biospheres prospects for long term survival. Figure 51 shows the fitness curves for the H3 biospheres in column 3 , and shows that such strong feedback, not possible in previous experiments where $T_{\text {ideal }}$ was further from $T_{0}$, is both possible and beneficial when initial conditions are close to the microbes ideal environment.

When $T_{\text {ideal }}=100$, the H3 systems in general have poorer survival rates than $\mathrm{H} 1$ systems despite performing better than H2 systems. With strong environmental feedback the H3 biospheres are more likely to be temperature limited than nutrient limited as the window of temperatures allowing for nutrient limitation shrinks as $\tau$ increases, meaning that the temperature is more likely to perform a random walk to either $T_{\text {lim }}^{+}$or $T_{\text {lim }}^{-}$and become 'stuck' there. This prevents the temperature from diverging far from $T_{0}$ as happens in the unconstrained H2 systems, however it also prevents temperatures from reaching values 

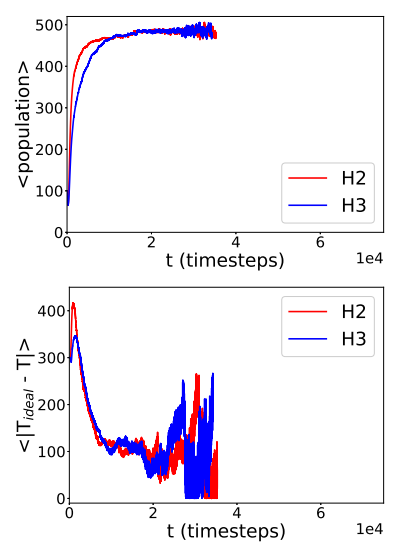

(a) $T_{\text {ideal }}=300 \tau=0.002$ interesting analysis.
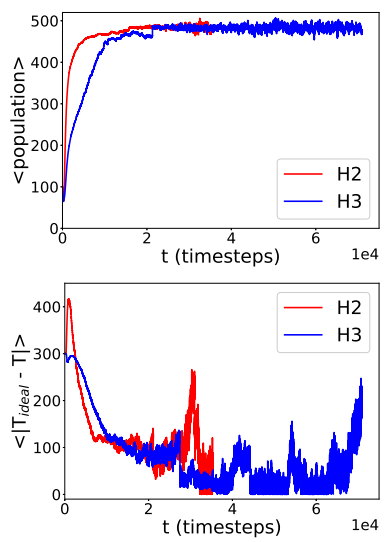

(b) $T_{\text {ideal }}=300 \tau=0.003$
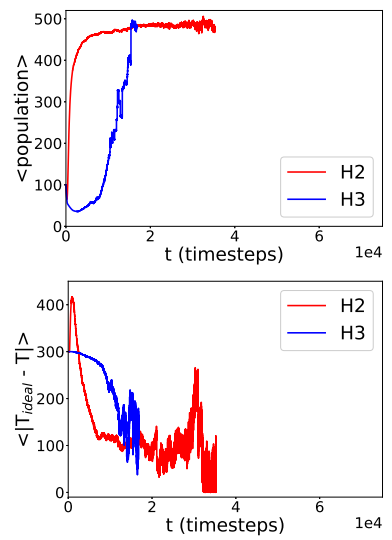

(c) $T_{\text {ideal }}=300 \tau=0.004$

Figure 6: Averaged population, and averaged $\left|T_{i d e a l}-T\right|$ of surviving H2 and H3 systems over time.

closer to $T_{\text {ideal }}$. As $T_{\text {lim }}^{-}<T_{0}$ must be true for a viable biosphere, the $P_{T}$ values of $\mathrm{H} 3$ systems in a temperature limited regime are greater than $P_{T, 0}$ and so in general H3 biospheres experience poorer survival rates than H1 biospheres when $T_{i d e a l}$ is close to $T_{0}$ even with strong feedback.

We compared how the average populations and temperatures of $\mathrm{H} 2$ and H3 systems behaved over time in extant systems. Figure 6 shows the average population and average $\left|T_{\text {ideal }}-T\right|$ over time, for those biospheres still alive at $\tau$ values differ slightly to those used in Figure 5 to show the impact of weak feedback, where H2 and H3 systems can behave very similarly, and because $\tau=0.005$ for $T_{\text {ideal }}=300$ results in biospheres going extinct too rapidly for

The top panel in Figure 6 shows that the population in H3 biospheres on average grows more slowly than in $\mathrm{H} 2$ biospheres. With feedback, as $T_{0} \neq T_{\text {ideal }}$, the growth rate of $\mathrm{H} 3$ microbes is initially slow compared to the temperature insensitive H2 microbes. The stronger the feedback on microbe metabolisms, 
the longer it will take for $\mathrm{H} 3$ biospheres to reach the environmental carrying capacity. With strong enough feedback H3 biospheres can remain in a temperature limited regime, instead of a resource limited regime - the case for all $\mathrm{H} 2$ biospheres.

With a lower reproduction rate, mutants are slower to appear in H3 biospheres, causing early temperature changes in the system to be slower than for H2 biospheres. The lower panels in Figure 6 show the average $\left|T_{\text {ideal }}-T\right|$ values for H3 and H2 extant systems over time. The H2 systems (in red) show an initial increase in this value, showing that many systems are degrading their environment. These systems will be short lived as they will have higher $P_{T}$ values, and quickly the average $\left|T_{i d e a l}-T\right|$ value drops, as those $\mathrm{H} 2$ biospheres that improve their environment survive via selection by survival. The H3 systems (in blue) do not show such a marked initial increase in $\left|T_{\text {ideal }}-T\right|$. With feedback, degrading H3 biospheres are self limiting. The early fitness of H3 biospheres is also lower than for the temperature insensitive $\mathrm{H} 2$ biospheres, and so H3 systems can get 'stuck' close to $T_{0}$ as new mutants take longer to appear.

Figure 7 shows the $P_{T}$ extinction values for $T_{\text {ideal }}=300$, with $\tau \in\{0.002$, $0.003,0.004\}$ for the H3 experiments. The constant null $P_{C}$ extinction probability is shown in black in each figure. Figure $7 \mathrm{a}$ shows the $\mathrm{H} 2$ experiments, and we see that early on, many $\mathrm{H} 2$ biospheres perturb their environment in a way that greatly increases their $P_{T}$ extinction probability (initially all have the value indicated by the black horizontal $P_{C}$ line). These systems however are short lived, and we see that over time, only those biospheres with smaller and smaller $P_{T}$ survive. Figures $7 \mathrm{~b}-7 \mathrm{~d}$ show $\mathrm{H} 3$ biospheres with increasing $\tau$. We see that with feedback to the microbes, the biospheres are unable to reach the high extinction probabilities reached in the $\mathrm{H} 2$ biospheres. As the microbes sensitivity increases, the H3 biospheres become less able to increase their $P_{T}$ values over the starting $P_{C}$ value, as doing so prevents their ability to consume nutrients resulting in starvation if conditions do not improve - thus 'anti-Gaian' dynamics are strongly self limiting when $\tau$ is high. This same feedback can hinder a H3 biosphere's ability to reach very low $P_{T}$ values and thus can also 




(a) H2 systems

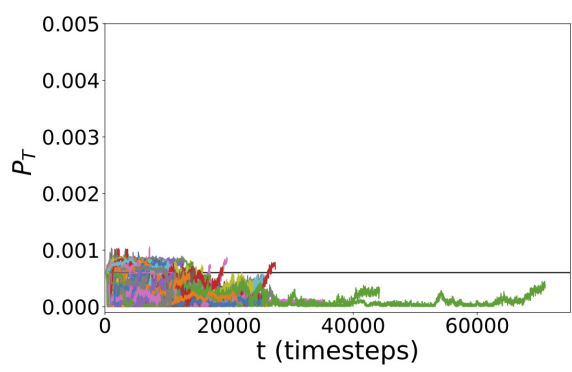

(c) H3 systems with $\tau=0.003$

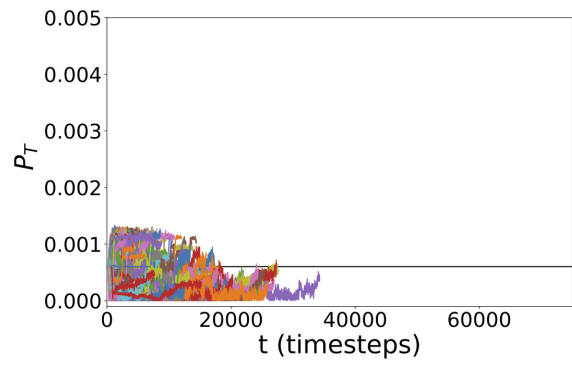

(b) H3 systems with $\tau=0.002$

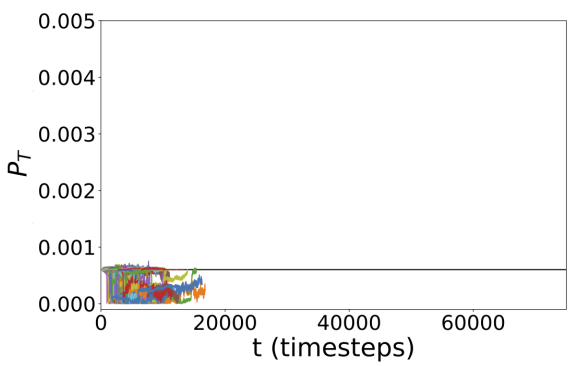

(d) H3 systems with $\tau=0.004$

Figure 7: Figures showing the $P_{T}$ values for $\mathrm{H} 2$ and $\mathrm{H} 3$ systems values for all experiments, with $T_{\text {ideal }}=300$. The $P_{C}$ constant extinction probability is shown in black in each figure.

hinder long term survival rates (Figure 7d).

\section{Discussion}

Demonstrating the efficacy of selection-by-survival is potentially important for understanding how ecosystems or biogeochemical cycles may 'evolve' 15, 16]. Our models suggest that selection by survival alone - the chance acquisition of beneficial adaptations - can promote long-term persistence of simple biospheres.

'Selection by survival' has been presented as a way to reconcile natural selection and the Gaia hypothesis without invoking selection for global homeostasis on the level of the biosphere. However, the absence of feedbacks between life and the abiotic environment means that in its simplest form it falls short of what is usually considered 'Gaia'. Our work shows that biospheres that incorporate environmental feedbacks on growth can additionally prevent 'anti-Gaian' 
dynamics from occurring and thereby further enhance their persistence. This situation supports the central idea of the Gaia hypothesis - namely that regulation can emerge from the interaction of life and the abiotic environment.

The role of feedbacks is strongly dependant on the early conditions of the system. Feedbacks can prevent young biospheres from expanding as rapidly as in their absence, but over longer time-spans surviving biospheres that include feedbacks can have significantly lower extinction probabilities than those without, depending on starting conditions. This means that early life attempting to become established on an inhospitable planet could be held back by environmental constraints, but in environments closer to ideal habitable conditions, feedbacks help to maintain that habitability. This result corresponds to the idea of 'Gaian-bottlenecks' [17] where early in a planet's history, the biosphere must quickly establish self-regulatory feedback mechanisms, or face extinction.

For 'randomly walking' systems, such as the models presented, the probability of reaching a point far from the starting position increases with the number of 'steps' taken - in our model, the number of mutations occurring in the biosphere. Our model systems start far from ideal conditions, and those whose random walk do not improve conditions have a high risk of extinction. Taken together, these points illustrate the importance of a guided random walk mechanism for the very long-term persistence of life on a planet.

Feedback between life and the environment is an inevitable feature of any biosphere, including the Earth. Furthermore, once a planet has abundant life it will inevitably become a significant driver of global biogeochemical cycles. We argue that both selection by survival and environmental feedback are likely to be important explanatory factors in any long-term persistence of life. The outstanding empirical challenge is to identify these two mechanisms amidst the complexity of Earth's biosphere and to resolve their relative contributions to the persistence of life on Earth - i.e. to determine whether Gaian regulation is a weak or strong stabilising force. 


\section{Acknowledgements}

We thank the Gaia Charity and the University of Exeter for their support of this work.

\section{Author Contributions}

Nicholson - Generated the data for the paper and co-wrote the paper.

Wilkinson - Initial paper concept and contributed to writing.

Williams - Provided feedback used to structure the paper, and suggested the creation and inclusion of Figure 7

Lenton - Co-wrote paper and suggested the creation and inclusion of Figure 1

\section{References}

口 [1] L. Margulis, J. E. Lovelock, Biological modulation of the earth's atmosphere, Icarus 21 (4) (1974) 471-489. doi:http://dx.doi.org/10.1016/ 0019-1035(74)90150-X.

URL http://www.sciencedirect.com/science/article/pii/ $001910357490150 \mathrm{X}$

[2] J. E. Lovelock, Gaia, a new look at earth, Oxford: Oxford University Press, 1979.

[3] W. F. Doolittle, Is nature really motherly?, Coevolution Quarterly 29 (1981) 58-63.

[4] R. Dawkins, The Extended Phenotype, Oxford University Press, 1982.

[5] T. M. Lenton, Gaia and natural selection, Nature 394 (6692) (1998) 439447.

URL http://dx.doi.org/10.1038/28792 
[6] T. Volk, Gaia's Body: Towards a Physiology of Earth, Springer New York, 1998.

[7] D. M. Wilkinson, Is gaia really conventional ecology?, Oikos 84 (1999) $533-536$.

[8] H. T. P. Williams, T. M. Lenton, The flask model: emergence of nutrientrecycling microbial ecosystems and their disruption by environmentaltering 'rebel' organisms, Oikos 116 (7) (2007) 1087-1105.

[9] H. T. Williams, T. M. Lenton, Environmental regulation in a network of simulated microbial ecosystems, PNAS 105 (30) (2008) 10432-10437.

[10] H. T. P. Williams, T. M. Lenton, Evolutionary regime shifts in simulated ecosystems, Oikos 119 (12) (2010) 1887-1899. doi:10.1111/j.1600-0706. $2010.18127 . \mathrm{x}$ URL http://dx.doi.org/10.1111/j.1600-0706.2010.18127.x

[11] T. M. Lenton, S. J. Daines, J. G. Dyke, A. E. Nicholson, D. M. Wilkinson, H. T. P. Williams, Selection for gaia across multiple scales, Trends in Ecology \& Evolution 33 (8) (2018) 633-645. doi:https://doi.org/10.1016/j.tree.2018.05.006. URL http://www.sciencedirect.com/science/article/pii/ S0169534718301186

[12] W. F. Doolittle, Natural selection through survival alone, and the possibility of gaia, Biology \& Philosophy 29 (3) (2014) 415-423. doi: 10.1007/s10539-013-9384-0

URL http://dx.doi.org/10.1007/s10539-013-9384-0

[13] J. Toman, J. Flegr, Stability-based sorting: The forgotten process behind (not only) biological evolution, Journal of Theoretical Biology 435 (2017) $29-41$.

[14] A. E. Nicholson, D. M. Wilkinson, H. T. P. Williams, T. M. Lenton, Multiple states of environmental regulation in well-mixed 
model biospheres, Journal of Theoretical Biology 414 (2017) 17-34. doi:http://dx.doi.org/10.1016/j.jtbi.2016.11.019

URL http://ww..sciencedirect.com/science/article/pii/ S0022519316303848

[15] F. Bouchard, Ecosystem evolution is about variation and persistence, not populations and reproduction, Biological Theory 9 (4) (2014) 382-391. doi:10.1007/s13752-014-0171-1. URL https://doi.org/10.1007/s13752-014-0171-1

[16] P. Bourrat, From survivors to replicators: evolution by natural selection 1. revisited, Biology \& Philosophy 29 (4) (2014) 517-538. doi:10.1007/ s10539-013-9383-1. URL https://doi.org/10.1007/s10539-013-9383-1

[17] A. Chopra, C. H. Lineweaver, The case for a gaian bottleneck: The biology of habitability, Astrobiology 16 (1) (2016) 7-22. 


\begin{abstract}
Alternative Mechanisms for Gaia - Appendix
Arwen E. Nicholson ${ }^{* 1}$, David M. Wilkinson ${ }^{2}$, Hywel T.P. Williams ${ }^{1}$, Timothy M. Lenton ${ }^{1}$

${ }^{1}$ Earth System Science, University of Exeter, Streatham Campus, Exeter, EX4 4 PY, UK

${ }^{2}$ School of Life Sciences, University of Lincoln, Joseph Banks Laboratories, Green Lane, Lincoln, LN6 7DL, UK
\end{abstract}

1. Model Description

The code used to generate the data for $\mathrm{H} 2$ and $\mathrm{H} 3$ systems is heavily based on a previous model called the Flask model [1, 2, 3, 4].

\title{
1.1. The Flask Environment
}

We have a single well mixed environment with no spatial element - we assume that in the flask the liquid medium is well mixed so that the composition of the flask is in a homogeneous steady state. The flask is characterised by nutrient levels and temperature. The nutrients present may be consumed by microbes and converted into biomass. The temperature is affected by and can affect, for H3 systems, the microbe activity.

The state of the flask is given by a vector V:

$$
V=\left(n_{1}, \ldots, n_{N}, T\right)
$$

where $n_{i}$ is the concentration of nutrient $i, N$ is the number of nutrients, and $T$ is the flask temperature.

As we break down each timestep into a number of iterations $n$ where $n$ is the total population of the system at the start of the timestep, we break down the inflow and outflow of the nutrient flask medium to prevent sudden changes at the the start of each timestep. The steps within a timestep would ideally all be computed in parallel but computational limitations prevent this, and so for agent based dynamics we effectively freeze the system while the selected microbes performs an action

*arwen.e.nicholson@gmail.com 
(being nutrient consumption / biomass production / reproduction / death). If we simply added and deducted the flow amounts at the start of each timestep, microbes selected at the beginning of a timestep could see a very different world to those selected at the end of a timestep if the population is large due to the microbes effect on the environment (nutrient consumption reducing nutrient levels and biomass creation affecting the abiotic parameters). Although these effects would largely average out due to the random selection of microbes during each timestep, a single large influx per timestep could be thought of as a periodic perturbation on the system which could affect the results seen. To counter this, we calculate the net influx of nutrients $N_{\text {net }}$ at the start of each timestep:

$$
N_{\text {net }}=I_{N}-O_{N} \times N_{\text {current }}
$$

where $I_{N}$ is the number of units of nutrient inflow per timestep, $O_{N}$ is the percentage outflow, and $N_{\text {current }}$ is the current nutrient levels in the system at the start of the timestep. We can then do $N_{\text {step }}=N_{\text {net }} / K_{\text {current }}$ where $K_{\text {current }}$ is the total population of the system at the start of the timestep, and then for each iteration within a timestep we increment the nutrient levels by $N_{\text {step }}$. This results in the same quantity of nutrients being added / removed from the system as if there was just one update at the start of the timestep, but it results in a much smoother transition and means that microbes selected at the start and end of a timestep will see much more similar worlds. In doing this, we treat nutrient levels as continuous but the microbes can only ever treat the nutrients as units. So while each iteration we might be adding 10.7 nutrient units per iteration, any microbes in the system can only act on the integer amounts of nutrients present.

We calculate the net temperature change due to diluting the current flask medium, by removing certain percentage $I_{T}$ of the existing flask medium and replacing it with the same volume of fresh influx at temperature $T_{\text {inflow }}$. So for the flask temperature we update each iteration by $T_{\text {net }}$ :

$$
T_{\text {net }}=T_{\text {inflow }} \times I_{T}-T \times I_{T}
$$

again each timestep we can then increment the flask temperature by $T_{\text {step }}=T_{\text {net }} / K_{\text {current }}$ where again $K_{\text {current }}$ is the total population of the system at the start of the timestep.

\subsection{Microbes}

The microbes consume and excrete nutrients in fixed proportions and affect the temperature of their environment as a side effect of biomass creation. The ratios of nutrient consumption / 
excretion and the byproduct effect on the temperature are genetically encoded for each microbe species. All microbes share the same preferred temperature $T_{\text {ideal }}$ (i.e. the temperature which results in the maximum growth rate). Microbes grow by consuming nutrients and converting them to biomass, and they reproduce asexually by splitting once their biomass reaches a threshold. Biomass is reduced by a fixed amount per timestep to represent the cost of staying alive. Microbes die if their biomass drops to a fixed threshold, which can happen during nutrient limitation or temperature limitation causing the microbes being unable to consume the nutrients present.

In the code we do not record microbes of the same species individually as doing so would slow the code considerably. Instead we group microbes of the same species together and record the species' total biomass. Thus each species can be thought of as a vector $S$ :

$$
S=\left(G, K_{S}, B, F, W, T_{\text {ideal }}\right)
$$

where $G$ is the species' genome (represented as a decimal number), $K_{S}$ is the population of the species, $B$ is the total biomass of the species, $F$ is total number of consumed food particles not yet converted into biomass, $W$ is the total number of waste particles not yet excreted by members of the species, and $T_{i d e a l}$ represents the temperature that maximise the growth for microbes in species $S$

The genotype $G$ of a microbe is recorded as the decimal representation of an 8 bit binary string, and this is used to group microbes into species. Microbes that share the same genome are of the same species. We create tables for microbe nutrient / excretion rules and abiotic effects and this genome is used as the reference to look up the particular metabolism rules for a microbe. With an 8 bit long binary genome there are 256 possible species (as each 'gene' in a genome can have the value 0 or 1$)$.

Microbes consume and excrete nutrients following genetically determined ratios. The nutrient ratios are fixed at the start of each simulation for each genome and remain constant. For example, with 4 nutrients: $a, b, c, d$, a microbe might need to consume nutrients with a ratio $\frac{1}{3}$ nutrient $a$ and $\frac{2}{3}$ nutrient $b$, and excrete a ratio of $\frac{1}{2}$ nutrient $c$, and $\frac{1}{2}$ nutrient $d$. This would be recorded in a vector as $\left[\frac{1}{3}, \frac{2}{3},-\frac{1}{2},-\frac{1}{2}\right]$. Positive values indicate that that nutrient is consumed, and negative that it is excreted by the microbe. We generate the look up tables for microbe metabolisms in the following way:

To generate these vectors for each genome, we start with 2 vectors of length $N$ where $N$ is the 
Table 1: Example microbe metabolism look up table with 4 nutrients $a, b, c$, and $d$

\begin{tabular}{ccccc}
\hline$G$ & $a$ & $b$ & $c$ & $d$ \\
\hline 0 & $1 / 2$ & $-1 / 3$ & $-2 / 3$ & $1 / 2$ \\
1 & $1 / 8$ & $3 / 8$ & $1 / 2$ & -1 \\
2 & $-1 / 5$ & $-3 / 5$ & 1 & $-1 / 5$ \\
\hline
\end{tabular}

number of nutrients. We populate these vectors with random numbers generated between $[-1,1]$ and then sum. For example if we had 4 nutrients, and our two vectors were $[-0.3,0.1,0.5,0.6]$ and $[-0.2,-0.2,0.1,-0.9]$ then summed we would have: $[-0.5,-0.1,0.6,-0.3]$. This would lead to the following ratios for consumption / excretion: $\left[-\frac{5}{9},-\frac{1}{9}, 1,-\frac{3}{9}\right]$. A microbe with this metabolism would only eat nutrient $c$ and would excrete nutrients $a, b$, and $d$. Not all metabolisms generated in this way will be viable. For example if the maximum possible number of nutrients a microbe can consume is $C^{\max }=10$ then the following metabolism $\left[\frac{5}{12}, \frac{7}{12},-\frac{1}{8},-\frac{7}{8}\right]$ would be unviable. This metabolism would require a microbe to consume 5 units of nutrient $a$ at the same time as 7 units of nutrient $b$, however this is never possible if $C^{\max }=10$. Units of nutrients are non divisible and can only be consumed in integer amounts. Thus any microbes with this metabolism would quickly starve to death. Generated metabolism vectors that result in all positive or all negative values are discarded, as microbes must both eat and excrete, and a new vector is generated for that genome. Table 1 shows an example look up table. To use Table 1, for a microbe with genome 000000010, we convert to its decimal value, 2 , and find that this microbe has metabolism where it consumes only nutrient $c$, and returns waste nutrients with the ratio $\frac{1}{5} a, \frac{3}{5} b$ and $\frac{1}{5} d$.

\subsubsection{Metabolism}

The microbes convert their food into biomass in an inefficient process that produces waste product. The efficiency of this conversion is given by $\theta$, and the amount of biomass produced is given by:

$$
B_{j}=\theta F_{j}
$$

where $B_{j}$ is the number of biomass units produced and $F_{j}$ is the number of food units currently 
'contained' with a microbe $j$. The waste excreted in this process is given by:

$$
W_{j}=(1-\theta) F_{j}
$$

where $W_{j}$ is the number of waste units produced, which are released into the environment after the biomass has been created, in the form determined by the microbe $j$ 's specific metabolism.

\subsubsection{Effect of temperature on metabolic rate}

The system temperature affects the rate at which microbes can consume nutrients which in turn affects the rate of biomass production and thus the growth of the microbes. A microbe will attempt to consume a maximum amount $C_{j}$ of nutrients each timestep with the demand being met depending on nutrient availability. The $C_{j}$ is calculated for each microbe $j$ as a function of the match between the microbes' genetically specified $T_{\text {ideal }}$ and the current temperature of the flask environment. This function has a Gaussian form and falls away smoothly from its maximum as the distance between the optimum and the current environment increases. Mathematically we write this as:

$$
\begin{gathered}
C_{j}=\psi_{j} C^{\max } \\
\psi_{j}=e^{-\left(\tau\left|T_{\text {ideal }}-T\right|\right)^{2}}
\end{gathered}
$$

where $C^{\max }$ is a constant determining the maximum possible rate of consumption for any microbe, $\psi_{j}$ is a microbe specific measure of the microbe's satisfaction with the current abiotic environment, $\tau$ is a universal constant parameter that determines how sensitive the microbes are to their environment ( $\tau=0$ means the microbes are not affected by the abiotic environment at all, and a higher $\tau$ means the microbes become more sensitive to the abiotic conditions). $p_{j}$ is a measure of the distance between the current temperature $\mathrm{T}$, and the microbes preferred temperature $T_{\text {ideal }}$. $\tau=0$ turns off any feedback from the environment to the microbes. This $\tau$ value is the only parameter that was changed between $\mathrm{H} 2$ and $\mathrm{H} 3$ systems. For all $\mathrm{H} 2$ systems $\tau=0$, for $\mathrm{H} 3$ systems $\tau>0$. 


\subsubsection{Effect of microbial activity on environment}

Microbes can affect the system temperature as a side effect of biomass creation. The effect the microbe has is proportional to its rate of biomass creation and thus its growth rate, so faster growing species will have a larger effect than slower growing species. Through the consumption of nutrients and excretion of waste products microbes also affect the nutrient levels in the environment.

Each microbe has an effect on the system temperature per unit of biomass created, and these effects are numbers in the range $[-1,1]$. These numbers are randomly generated in this range at the beginning of each simulation for each species and remains constant throughout the simulation. Thus each member of a species has the same effect on the system temperature for the duration of the simulation.

\subsubsection{Maintenance Cost}

There is a fixed biomass cost $\lambda$ of staying alive for each microbe. This reduces a microbe's biomass by a constant rate. This cost represents the energy costs of maintaining cellular machinery and metabolic inefficiency. This cost is assumed to be lost as unrecoverable heat radiation. This ensures that the chemicals cannot be infinitely recycled and it sets the carrying capacity of the system. This carry capacity is reached when the total heat dissipation matches the energy supplied in the form of chemicals, i.e. the food the microbes consume. $\lambda$ is identical for all species.

\subsubsection{Reproduction and Mutation}

If the microbe is able to consume enough chemicals to reach the reproduction threshold $T_{R}$, it will reproduce asexually, splitting in half. Half of the biomass with go to the new microbe and the parent microbe will be left with half its biomass. The new microbe will have the same genome as the parent unless a mutation occurred during the reproduction. There is a small constant probability of mutation, $P_{m u t}$, for each locus. During a reproduction event, the code iterates through the genome of the new microbe and if a mutation occurs at a locus then the gene at that point will be 'flipped', turning it to 0 if it were previously 1 , or to 1 if it were previously 0 . This new mutant genome will then dictate the new microbe's metabolism.

\subsubsection{Death}

If a microbe's biomass falls to a starvation threshold $B_{D}$ the microbe will starve to death. There is another small probability of death $D$ that represents death by hazardous mutation or damaging 
local environmental changes etc. When a microbe dies its biomass is removed from the system, as if the dead microbe, for example, fell to the bottom of the ocean. During a death event, we first check to see if the selected microbe has enough biomass to avoid death by starvation. If the microbe has not starved to death it will be killed with probability $D$.

\subsection{Selecting a microbe}

We use agent based dynamics in our model. This means within a timestep, a microbe is chosen randomly for an event and time is effectively frozen while the microbe performs that event. Time is then restarted and another microbe is chosen at random for an event.

As we record microbes grouped together in a species (Equation 4), for any particular species we have the population of the species, the total species biomass, and the total consumed food not yet converted into biomass. To select a single individual of a particular species we therefore need to determine how much biomass and unconverted food this individual has. If a microbe is selected for a reproduction event, we need to know how much biomass it has to know if it has reached the reproduction threshold for example.

There will be variation between individuals of a species and so we assume a normal distribution of biomass and unconverted food between individuals of a species. The biomass normal distribution is centred around the average amount of biomass $B_{a v}$ per microbe (i.e. the total species biomass divided by the species population), with standard deviation of the distribution is $B_{a v} \times 0.1$. The normal distribution for the unconverted food is the same but with $F_{a v}$, the average amount of unconverted food per microbe, instead. The standard deviation for both distributions is small, resulting in a small level of variation in the population. Therefore most individuals of the same species will have the same biomass and food levels.

Once we have selected a microbe and calculated its biomass and food level, the microbe can then attempt to perform the event it was selected for.

\section{Biosphere-wide extinction probabilities}

The probabilities of biosphere-wide extinction are determined in the following way:

\subsection{H1 systems}

For the null model we assume a constant extinction probability for each biosphere at each model timestep, resulting in exponential decay in the number of surviving biospheres. The probability of 
extinction for all time for $\mathrm{H} 1$ systems is a constant:

$$
P_{C}=C
$$

We set $C$ to have the same value as the starting extinction values for the selection by survival, and the added feedback experiments.

\subsection{H2 and H3 systems}

For our $\mathrm{H} 2$ and $\mathrm{H} 3$ systems, the flask temperature impacts the biosphere-wide probability of extinction:

$$
P_{T}=a+b \times\left|T_{\text {ideal }}-T\right|
$$

$T$ is the system temperature, and depends on the genetic makeup of the microbe community currently alive in the system, $a=2 \times 10^{-6}$ is a constant background probability of global extinction, and $b=2 \times 10^{-6}$ controls the impact the flask temperature has on the probability of extinction. Here $T_{i d e a l}$ - the temperature for which microbes have the highest fitness is also the flask temperature that results in the lowest probability of flask extinction.

\section{Parameters}

The parameters used for the flask systems presented in the paper. 


\begin{tabular}{|c|c|c|}
\hline Parameter & Value & Description \\
\hline$N$ & 4 & Number of nutrients \\
\hline$B_{R}$ & 120 & Reproduction threshold (biomass units) \\
\hline$B_{D}$ & 50 & Starvation threshold (biomass units) \\
\hline$P_{m u t}$ & 0.01 & $\begin{array}{l}\text { Probability of mutation at each locus during repro- } \\
\text { duction }\end{array}$ \\
\hline$D$ & 0.002 & $\begin{array}{l}\text { Probability of death by natural causes (other than } \\
\text { starvation) at each timestep }\end{array}$ \\
\hline$\lambda$ & 1 & Maintenance cost (biomass units / timestep) \\
\hline$\theta$ & 0.6 & Nutrient conversion efficiency \\
\hline$C^{\max }$ & 10 & $\begin{array}{l}\text { Maximum number of nutrients a microbe can con- } \\
\text { sume in any single event }\end{array}$ \\
\hline$\tau$ & $\begin{array}{lll}\{0.002, & 0.0025, & 0.003 \\
0.004, & 0.005, & 0.007 \\
0.009\} & & \end{array}$ & $\begin{array}{l}\text { Level of influence of abiotic environment on } \\
\text { metabolism }\end{array}$ \\
\hline$\mu$ & {$[-1,1]$} & $\begin{array}{l}\text { The impact a microbe has on the flask temperature } \\
\text { per biomass created is taken from this range. }\end{array}$ \\
\hline$I_{N}$ & 150 & Rate of nutrient influx (units / timestep) \\
\hline$O_{N}$ & 0.25 & Rate of nutrient outflux (percentage / timestep) \\
\hline$I_{T}$ & 0.2 & $\begin{array}{l}\text { Percentage of flask medium replaced with fresh influx } \\
\text { each timestep, used for calculating the flask temper- } \\
\text { ature change (percentage / timestep) }\end{array}$ \\
\hline$K_{M}$ & 100 & Number of individuals in flask inoculum \\
\hline$t_{\text {prep }}$ & 500 & Flask equilibration time prior to seeding (timesteps) \\
\hline$T_{\text {ideal }}$ & $\{500,300,100\}$ & $\begin{array}{l}\text { The temperature at which the biosphere-wide extinc- } \\
\text { tion probabilities are at a minimum, and the univer- } \\
\text { sal microbe temperature preference }\end{array}$ \\
\hline$T_{\text {inflow }}$ & 0 & $\begin{array}{l}\text { Environmental temperature in the absence of mi- } \\
\text { crobe activity }\end{array}$ \\
\hline
\end{tabular}




\section{Method}

\subsection{H1 systems}

For the null model we simply apply the biosphere-wide extinction probability $P_{C}$ to $10^{4} \mathrm{H} 1$ systems until all are extinct. This is an exponential decay.

\subsection{H2 and H3 systems}

We again run $10^{4}$ experiments for each scenario. In each experiment we seed the flask with a single species, and we fix this species to have $\alpha=0$ impact on the flask temperature per biomass created. This means all flask systems start with identical starting conditions, and any differences in flask experiments is due to the mutants arising in the system, and not due to differing starting configurations.

We use agent based dynamics to run the $\mathrm{H} 2$ and $\mathrm{H} 3$ simulations. A timestep is broken down into iterations, the number of iterations matches $n$ the number of microbes alive in the system at the start of the timestep. For each iteration we perform the following steps:

- We run the inflow and outflow of the nutrient rich flask medium for $10^{4}$ timsteps to reach an equilibrium state before seeding

- Seed with 100 microbes of the same species with $\alpha=0$ impact on the flask temperature per biomass created

- For each iteration we perform the following steps:

- Influx / outflux of flask medium (at constant temperature) and nutrients via trickle

- An individual is selected randomly for a death event

- An individual is selected randomly for a nutrient consumption event

- An individual is selected randomly for a biomass creation event

- An individual is selected randomly for a reproduction event

- We repeat this process $n$ times for one timestep.

- Each timestep, the flask system has the temperature dependant probability $P_{T}$ of going extinct.

- Each simulation is run until the system goes extinct. 


\section{References}

[1] H. T. P. Williams, T. M. Lenton, The flask model: emergence of nutrient-recycling microbial ecosystems and their disruption by environment-altering 'rebel' organisms, Oikos 116 (7) (2007) $1087-1105$.

[2] H. T. Williams, T. M. Lenton, Environmental regulation in a network of simulated microbial ecosystems, PNAS 105 (30) (2008) 10432-10437.

[3] H. T. P. Williams, T. M. Lenton, Evolutionary regime shifts in simulated ecosystems, Oikos 119 (12) (2010) 1887-1899. doi:10.1111/j.1600-0706.2010.18127.x

URL http://dx.doi.org/10.1111/j.1600-0706.2010.18127.x

[4] A. E. Nicholson, D. M. Wilkinson, H. T. P. Williams, T. M. Lenton, Multiple states of environmental regulation in well-mixed model biospheres, Journal of Theoretical Biology 414 (2017) 17-34. doi:http://dx.doi.org/10.1016/j.jtbi.2016.11.019 URL http://www.sciencedirect.com/science/article/pii/S0022519316303848 